\title{
Research on Networked Sliding Mode Predictive Controller Based on Particle Swarm Optimization
}

\author{
Yang Yin*, Hongke Li and Zhengying Ren \\ College of Electrical Engineering, Naval University of Engineering, Wuhan, China \\ ${ }^{*}$ Corresponding author
}

\begin{abstract}
Aiming at the development of ship information and intellectualization, this paper researched the control law design problems of the network control system under total ship computing environment (TSCE). Firstly, we analyzed and constructed the computing environment network architecture for the development of ship intelligent information technology. Then, the sliding mode predictive control law based on particle swarm optimization was designed for the delay and packet loss problem caused by information network. The control strategy and realization method were analyzed, and the actual control quantity selected at each moment was the optimal sliding mode prediction control signal provided by the actuator. The simulation results showed the effectiveness and robustness of the proposed algorithm.
\end{abstract}

Keywords-Total Ship Computing Environment (TSCE); Networked Control Systems (NCS); Sliding Mode Predictive Control; Particle Swarm Optimization Algorithm (PSO)

\section{INTRODUCTION}

With the development of intelligent technology of new ship information technology, the research of integrated platform system with interconnection, intercommunication and interoperation is getting more and more attention. Intelligent ship total ship computing environment (TSCE) is a new generation of shipboard integrated system which is leading changes in design and integration mode of ship system [1]. Replacing the original independent control system with network control, make numerous such as sensors, actuators and controllers are connected through the network, can make the ship's architecture flattening and greatly improve the level of ship automatic control and intelligent management. However, problems such as the delay of the network itself, packet loss, communication constraints and multi-packet transmission will inevitably lead to the change of system control strategy, namely, Networked control systems (NCS) [2-5]. Therefore, it is of great practical significance to study the control technology under the TSCE network. This paper focuses on the ship TSCE system structure and its control network system optimization. Inspired by the discrete sliding mode predictive control method [6], we design the sliding mode predictive controller based on particle swarm optimization [7,8].And then study the control strategy and the implementation method, in order to reduce the influence of network on the control system performance and guarantee system robustness under external disturbance.

\section{TSCE ARCHITECTURE AND NETWORK CONTROL MODELING}

The TSCE has three tiers: Core, Adaptation, and Presentation. The Core Tier provides a common environment hosting the majority of the software applications on a redundant infrastructure whose targeted hardware location is designed to be transparent to the applications. The Adaptation Tier utilizes much more compact hardware to provide a means to integrate software into the TSCE. The Presentation Tier is that part of the TSCE responsible for rendering displays on the consoles. Its control hierarchy network configuration is shown in Figure I.

DCU is distributed control units. DAP is Distributed Adaptation Processors which perform function of controller and interface to other applications running in the TSCE infrastructure. RTU is remote terminal units that work as the interface to the engineering plant equipment, just like Soft PLC application and Ladder Logic to control and drive the remotely located I/O, and so on. HCI is Human Computer Interface.

Mainstream industrial Ethernet technologies include PNO Profinet, EPSG Powerlink, ODVA Ethernet/IP, ETG EtherCAT and EPA. EPA is the first field bus national standard with independent intellectual property rights in China. Regardless of which network technology is chosen, the network problem of delay packet loss is brought to the control system.

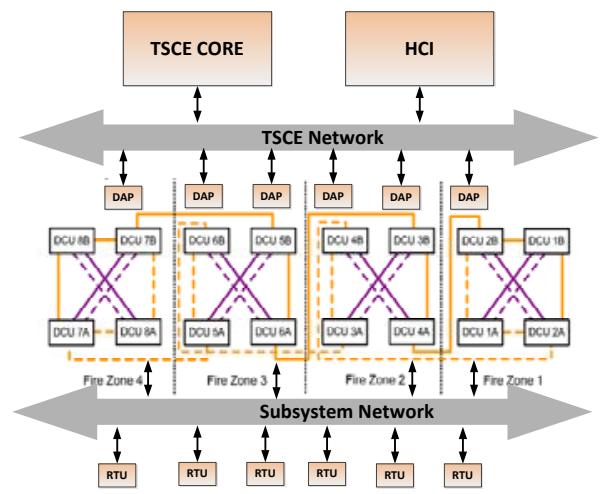

FIGURE I. TSCE CONTROL HIERARCHY NETWORK CONFIGURATION

Consider the discretization model of a class of network control systems under TSCE.

$$
x(k+1)=A x(k)+B u_{a}(k)+G \cdot M(k)
$$


Where ${ }^{x(k)}$ is the state, $M(k)$ is expressed as an external disturbance. $U_{a}$ represents the input control amount of the system executor. Because the introduction of TSCE network, the controller and actuator information transmission may be time delay or packet loss. So some moments actuator may receive less information than the controller transmission. Thus cause control performance degradation and even lead to instability. This paper intends to use the sliding model predictive controller based on particle swarm to produce multi-step input predictive control values. And then set buffer zone in front of actuators, storing the predictive control values. According to the actual network conditions to select the latest optimizing control values control the controlled object. The typical network control system model block diagram is shown in Figure II.

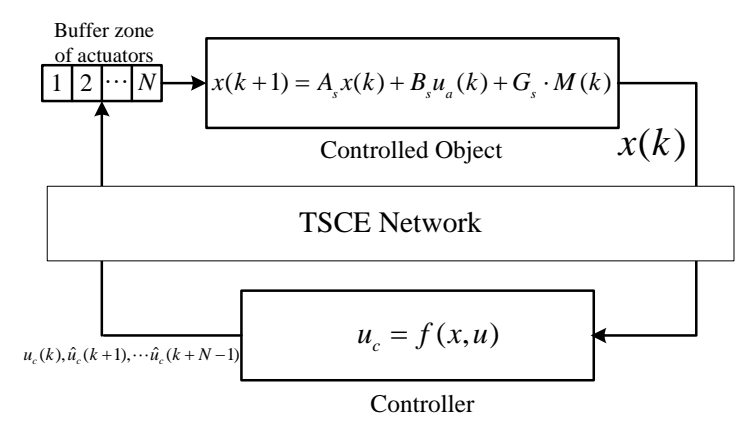

FIGURE II. BLOCK DIAGRAM OF TSCE NETWORKED SYSTEM

\section{Predictive Control of Network SLiding Mode BASED ON PARTICLE SWARM OPTIMIZATION}

The traditional design of discrete variable structure control method usually use uncertain bound as the prerequisite to ensure the robust stability of closed-loop system. Due to consider condition is the worst, cause controller is too conservative and chattering. In order to solve this problem, a discrete sliding mode prediction controller based on particle swarm optimization is designed. After the controller receives the sensor signal at current moment, the discrete sliding mode prediction algorithm with particle swarm optimization is used to generate certain predictive steps control signal.

\section{A. Discrete Sliding Mode Predictive Control}

Firstly, we give the analysis and modeling of the control algorithm of sliding mode prediction function. Consider the system (1) and define the linear sliding mode function.

$$
s(k)=C x(k)
$$

The ideal sliding mode must satisfy the motion equation of the following system:

$$
\left\{\begin{array}{l}
x(k+1)=A_{s} x(k)+B_{s} u_{a}(k) \\
C x(k)=0
\end{array}\right.
$$

The sliding surface is determined by quadratic optimal control or pole assignment. Regardless the presence of parameter perturbation and external disturbance or not, the movement of the system starting from any initial state can be reached in neighborhood of the sliding mode surface within limited time and stability. Then act into a quasi sliding mode motion.

The nominal model of system (1) is used as the prediction model.

$$
x(k+1)=A_{s} x(k)+B_{s} u_{a}(k)
$$

The predicted time domain is $N$ and the prediction value of the sliding mode function of the future $\mathrm{P}$ step is

$$
s_{y}(k+p)=C A^{p} x(k)+\sum_{i=1}^{p} C A^{i-1} B u(k+p-i)
$$

Using the error between the current measured value and the predicted value, the predicted value of the future time is corrected as:

$$
\begin{array}{r}
\hat{s}_{y}(k+p)=s_{y}(k+p)+H_{p}\left[s(k)-s_{y}(k \mid k-p)\right] \\
=C A^{p} x(k)+\sum_{i=1}^{p} C A^{i-1} B u(k+p-i)+H_{p}\left[s(k)-s_{y}(k \mid k-p)\right]
\end{array}
$$

Where $s_{y}(k \mid k-p)$ Denotes the predicted $k$ step value of the sliding mode at time $k-p$. The correction factor is $h_{p} \in R$, usually choose $h_{1}=1$.

$$
s_{y}(k \mid k-p)=C A^{p} x(k-p)+\sum_{i=1}^{p} C A^{i-1} B u(k-i)
$$

Then we select the optimization index:

$$
J_{E}=\sum_{p=1}^{N} q_{p}\left[\hat{s}_{y}(k+p)-s_{r}(k+p)\right]^{2}+\sum_{p=1}^{N} r_{p}[u(k+p-1)]^{2}
$$

Where $q_{p}, r_{p}$ are the weighted coefficients, $s_{r}(k+p)$ is sliding mode reference trajectory.

The reference trajectory is a desired trajectory. The selection of sliding mode reference trajectory determines the final form of the variable structure control law obtained. Therefore, we select the most commonly used index approach rate as the sliding mode reference trajectory. 


$$
\left\{\begin{array}{l}
s_{r}(k+p)=(1-q T) s_{r}(k+p-1)-\varepsilon T \operatorname{sgn}\left(s_{r}(k+p)\right) \\
s_{r}(k)=s(k)
\end{array}\right.
$$

Where $\varepsilon>0, q>0,1-q T>0$.

Control problem is transformed into search $u^{*}(k)$ to makes the performance index $J_{E}$ optimum. According to the Eq.6, the input and state of the past and present time can pass out the input of the future time. Then rolling optimization, find out the optimal control input. In this paper, particle swarm optimization algorithm is proposed to solve this optimization problem.

\section{B. Particle Swarm Optimization}

Particle swarm optimization (pso) is an evolutionary algorithm based on group bionics, which mainly uses information sharing and group evolution mechanism in biological groups. In recent years, particle swarm algorithm has been developed rapidly and successfully applied to various optimization problems.

The basic idea of particle swarm algorithm is as follows: the solution of optimization problem is regarded as a point ( $d$ dimension particle) in the search space. Several particles constitute a group. The pros and cons of each particle performance depends on fitness value of objective function of optimization problem. The particle velocity determine the direction and speed of flight. All particles follow the current optimum particles search in the solution space. The algorithm firstly initializes a group of random particles (candidate solution) and then finds the optimal solution by gradual iteration. Each particle represents a candidate solution, and the position and velocity relation of the particle can be described as:

$$
v_{i k}(t+1)=w v_{i k}(t)+c_{1} r_{1}\left(p_{i k}(t)-x_{i k}(t)\right)+c_{2} r_{2}\left(p_{g k}(t)-x_{i k}(t)\right)
$$

$$
x_{i k}(t+1)=x_{i k}(t)+v_{i k}(t+1)
$$

Where $k_{\text {is Pointer of iterations, }} x_{k}$ is current position of particle at iteration $k, v_{k}$ is the velocity of particle at iteration $k, c_{1}, c_{2}$ is acceleration constant, $w_{\text {is }}$ inertia weight factor. $P_{i}=\left(p_{i 1}, p_{i 2}, \cdots p_{i d}\right)$ represents the individual extreme value, $P_{g}=\left(p_{g 1}, p_{g 2}, \cdots p_{g d}\right)$ represents the group global extreme value.

In the iterative process, the particle can update its own speed and position by tracking individual extremum and group global extreme values. Formula (10) mainly calculates the new velocity of the first particle through three parts. First part is the velocity of the first particle at the first moment. The second is the distance between the current position and its best position. The third is the distance between the current position and the best position of the group. The particle swarm algorithm has less parameters, simple operation, and the initial convergence performance is excellent.

\section{IMPLEMENTATION FOR PSO OPTIMIZATION SLIDING MODE PREDICTION CONTROL}

Because the standard particle swarm algorithm is easy to get into local optimum, and combined with TSCE network characteristics, this paper selects the specific implementation steps of particle swarm prediction control algorithm.

Step 1: Particle swarms initialize. Identify TSCE network delay, set the population size. Then set the upper and lower limit of the search space, limit the maximum speed and minimum speed of the particle, and initialize the position and velocity of the particle;

Step 2: The fitness of the particle is calculated. The optimization objective function formula (9) of the particle is calculated so that the formula (8) is satisfied.

Step 3: Calculate individual extremum and global extremum. Initializes the initial individual extreme point ${ }^{P_{i}}$ of each particle and sets it to the initial position. The best setting in $P_{i}$ is set as the fitness value $P_{g}$ of the initial global extreme point. Then the individual extremum and global extremum of each step are updated successively.

Step 4: The dynamic shrinkage factor method is selected to adjust the weight coefficient to avoid getting into local optimum.

$$
w(k)=w_{\min }+\frac{k_{\max }-t}{k_{\max }}\left(w_{\max }-w_{\min }\right)
$$

Where $k_{\max }$ is maxima pointer of iterations, $w_{\max }$ is maxima inertia weight factor, $w_{\text {min }}$ is minimum inertia weight factor, $v_{k}$ is Velocity of particle at iteration $k$.

Step 5: According to Eq.9, Eq.10, Eq.10, adjust the position and velocity of the particle.

Step 6: Check the termination condition: when the maximum number of iterations is reached or the desired precision is reached, the iteration is terminated, otherwise the step 2 is returned. The optimal value $u^{*}(k), u^{*}(k+1), \cdots$, $u^{*}(k+N-1)$ of the group control quantity of the time is stored in the actuator end buffer after network transmission. According to the situation of network actual delay packet loss, the corresponding control actuator is selected to realize the control of TSCE control system.

\section{SIMULATION EXAMPLE}

Suppose a ship TSCE network control system is modeled as equation (1). The nominal parameters are as follows: 


$$
\left\{\begin{array}{c}
x(k+1)=\left[\begin{array}{ccc}
1 & 0.08198 & 0.0005247 \\
0 & 0.6412 & 0.004486 \\
0 & -3.068 & -0.02147
\end{array}\right] x(k)+\left[\begin{array}{c}
-0.449 \\
-8.943 \\
-76.46
\end{array}\right] u_{a}(k)+M(k) \\
y(k)=\left[\begin{array}{lll}
1 & 0 & 0
\end{array}\right] x(k)
\end{array}\right.
$$

Where the Uncertainty is

$$
M(k)=\sigma \%\left[\begin{array}{lll}
0 & 1 & 1
\end{array}\right]^{T}[1+\sin (3 \pi k / 20)]
$$

Take the sliding mode surface parameter as $C=\left[\begin{array}{lll}47 & 12 & 1\end{array}\right]$ and approach the law parameter as $q=5$, $\varepsilon=1$. Q $R 、 H=\operatorname{diag}[1,1,1], \sigma \%$ is the percentage of interference. The predicted number of steps is greater than or equal to the maximum time delay period of the actual network. Networked sliding mode predictive controller based on particle swarm optimization design is adopted, and the performance of the system is analyzed. The unit step response of the system is shown in the red real curve in Figure III when the system has five percent constant value disturbance and sinusoidal disturbance. The response curve of the system with fifteen percent constant value disturbance and sinusoidal perturbation is shown in Figure VI. In order to facilitate comparison, the virtual curve represents the step response curve of the system with the traditional pole assignment control algorithm and without PSO optimization.

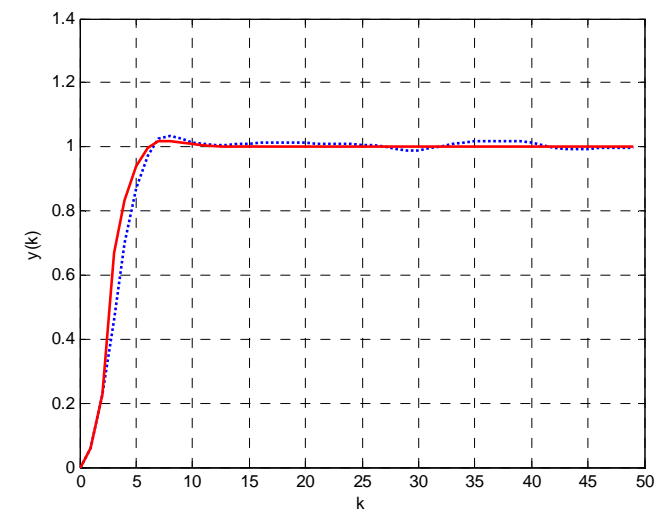

FIGURE III. SIMULATION RESULTS WITH FIVE PERCENT INTERFERENCE

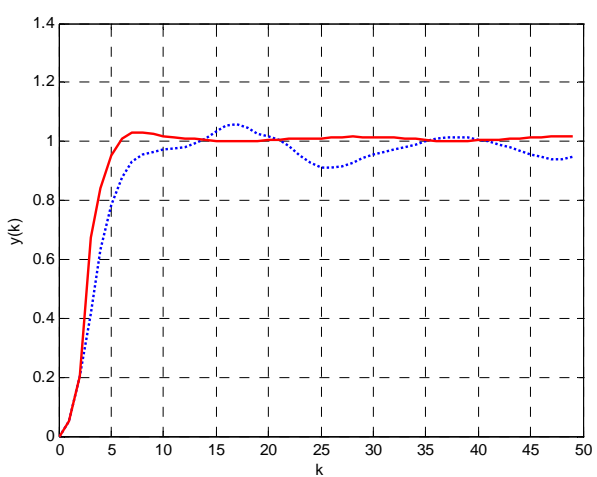

\section{FIGURE IV. SIMULATION RESULTS WITH FIFTEEN PERCENT INTERFERENCE}

The simulation results show that, when the system interference is less and due to the influence of the network, the traditional method has a slow response speed and a certain steady-state error. When the interference of the system increases, the traditional method can not track input effectively, and there is a possibility of large oscillation. In contrast, using networked sliding mode predictive controller based on particle swarm optimization, The system has good dynamic and steady state performance. And it has strong robustness against external disturbance and certain network delay.

\section{SUMMARY}

According to the trend of intellectualized development of ship information, this paper studied the network control problem of the system under ship's TSCE network. Based on particle swarm optimization, a sliding mode prediction control strategy was designed and its concrete realization steps were given. The simulation results showed that the proposed algorithm was reasonable and effective. Next, we will continue to study the method of combining the characteristics of TSCE network with the control theory to improve the performance of the ship control systems.

\section{REFERENCES}

[1] DONG Xiao-ming, SHI Chao-ming, etc. Analysis on the Architecture of USN DDG-1000 Total Ship Computing Environment. Chinese Journal of Ship Research. 2012, 7(6), 7-15.

[2] Liu, Y.-C \& Liu, B \& Zhang, Y \& Li, W.-G. Stability analysis for networked control systems with bilateral random delay and packet dropout. Control and Decision. 2017,32,1565-1573.

[3] Xie, R \& Fan, W \& Wang, C \& Chen, Q. Dead band scheduling and control co-design method for uncertain networked control systems. 2016,40, 643-652.

[4] J.Baillieual and Panos J.Antsaklis. Control and communication challenges in networked real-time system. Proc. IEEE, vol.95, no.1, Jan.2007, 9-28.

[5] Li Hong-bo, SUN Zeng-qi, SUN Fu-chun. Networked control systems: an overview of state-of-the-art and the prospect in future research. Control Theory \& Applications, 2010, Vol. 27, No.2, pp.238-243.

[6] Song lizhong, Chen shaochang, Yao qionghui. Predictive discrete variable structure control [J]. Control theory and application, 2004, 21(5), 826-829.

[7] DAI Yong-bin. Analysis and Simulation Research on Nonlinear Predictive Control Based on Particle Swarm Optimization. Journal of 
Liaoning University of Technology (Natural Science Edition). Vol.34, No.6, Dec.2014:360-363.

[8] Gao peng, liu haoran, hao xiaochen, et al. Research on predictive control of hybrid model based on particle swarm optimization [J]. Mechanical and electrical engineering, 2012, 28(2): 231-234. 